Correa M.G., Arroyo P., Mourgues C., and Flager F. (2017). "Comparing Choosing by Advantages and Weighting, Rating and Calculating Results in Large Design Spaces.” In: LC3 2017 Volume II - Proceedings of the 25th Annual Conference of the International Group for Lean Construction (IGLC), Walsh, K., Sacks, R., Brilakis, I. (eds.), Heraklion, Greece, pp. 259-266. DOI:https://doi.org/10.24928/2017/0248

\title{
COMPARING CHOOSING BY ADVANTAGES AND WEIGHTING, RATING AND CALCULATING RESULTS IN LARGE DESIGN SPACES
}

\author{
María Gabriela Correa ${ }^{1}, \mathrm{Paz}_{\text {Arroyo }}{ }^{2}$, Claudio Mourgues ${ }^{3}$, and Forest Flager ${ }^{4}$
}

\begin{abstract}
Architecture, Engineering and Construction (AEC) projects are complex systems that are evaluated based on many factors. Multi-Criteria Decision Making (MCDM) methods are used to support AEC project teams in this process. Traditionally, these decisions are made using the Weighting, Rating and Calculating (WRC) method. Recent literature shows benefits of the Choosing By Advantages (CBA) method compared to WRC. However, these studies have been made in the context of comparing and ranking a small number of design alternatives (2-10). This research presents a case study in which CBA and WRC are applied to a large design space. The results show that CBA allowed for a more complete comparison of design alternatives. In addition, CBA enabled decision makers to explicitly evaluate performance versus cost, which led to more transparent and Pareto optimal decisions considering all alternatives in the design space.
\end{abstract}

Keywords: Choosing By Advantages, CBA, WRC, Large Design Spaces, Multi-Disciplinary Optimization.

\section{INTRODUCTION}

Contemporary buildings are complex systems that are evaluated based on multiple performance criteria including energy consumption, acoustical performance, thermal occupant comfort, indoor air quality, cost and many other issues (Hopfe et al. 2013). Building design involve the collaboration of many specialists. Typically these specialists are organized by discipline, with each discipline being responsible for the design of a component (subsystem) of the overall system (Balling and Rawlings 2000).

Specialists strive to meet design goals and constraints that are particular to the specific subsystem they are designing. The goals of the individual subsystems can conflict, i.e. an optimal design from the perspective of one subsystem may not be optimal or even feasible from the perspective of other subsystems (Tappeta and Renaud 2001). In addition, subsystems are often coupled, which means that design decisions in one subsystem may influence or even control design decisions in the coupled subsystem.

Multi-Criteria Decision Making (MCDM) methods are used to help project teams to choose the best alternative for the decision maker considering multiple factors. Weighting, Rating and Calculating (WRC), a MCDM method, is widely used in the industry (Arroyo

$1 \quad$ Master Student, Construction Engineering and Management Dept., School of Engineering, Pontificia Universidad Católica de Chile, Santiago, Chile, +569 56189350, mgcorrea1@uc.cl

2 Assistant Professor, Pontificia Universidad Católica de Chile, Santiago, Chile, +56 22354-4244, parroyo@ing.puc.cl

3 Assistant Professor, Pontificia Universidad Católica de Chile, Santiago, Chile, +56 22354-4244, cmourgue@ing.puc.cl

4 Research Associate and Lecturer, Department of Civil and Environmental Engineering, Stanford University, Califoria, United States, +1.415.728.7197, forest@standford.edu 
et al. 2016). However, several authors have proved that Choosing by Advantages (CBA) presents several benefits over WRC (Arroyo et al. 2016; Arroyo et al. 2014) in terms of transparency and its capacity to reach consensus. CBA presents many benefits in the decision-making process but these benefits have been proven only in scenarios that consider a relatively small number of alternatives (2-10).

Multidisciplinary Design Optimization (MDO) methods formalize problem decomposition and coordination among groups working on the design of complex systems (AIAA 1991). These systematic procedures leverage computer processing power to enable design team to explore orders of magnitude more alternatives than possible using conventional methods (Vandenbrande, Grandine, et al. 2006). Potential benefits include compressing design cycle time, evaluating many more potential design alternatives and yielding substantial product quality and performance gains.

The comparisons that a decision maker should make to assess thousands of alternatives using CBA in a design process that uses MDO methods to generate alternatives would be overwhelming. Therefore, we developed a protocol to use CBA in this context. This study compares the results of using of using CBA and WRC in a large design space.

\section{BACKGROUND}

\subsection{Multi-Criteria Decision Making Methods}

During the design process, decision makers must decide between a set of design alternatives, which could involve multiple criteria. In those cases, MCDM methods, are tools to collaborate in the decision making process. Many MCDM methods exist. However value-based methods, such as WRC, are widely used in the AEC industry (Arroyo et al. 2016; Arroyo et al. 2015).

WRC is a method that weights factors, that are elements or components of a decision (e.g., energy usage, acoustic performance, and air quality), to decide which is the best alternative. This method uses three principal steps: (1) decision makers weights factors considered in the decision; (2) they rate the alternatives for each factor in function of the attributes (or characteristic) that each alternative has; and (3) the weight of each factor multiplies each alternative as a function of the rate. The weighted sum for each alternative gives the final score for that alternative. The alternative that has highest final score is the alternative that offers more benefits for the decision maker.

CBA is a method developed by Suhr (1999), that is based on the advantages of each alternatives and not on the weight of factors considered in the decision.

CBA method has eight steps: (1) identify alternatives; (2) identify factors; (3) define the criteria to assess each factor; (4) determinate the attributes that characterize each alternative; (5) decide the advantages of each alternative; (6) decide the Importance of Advantage (IoA) for each alternative; (7) Sum the IoA for each alternative; (8) decide the best alternative in function of the IoA and cost of each alternative.

Previous research has demonstrated some benefits of CBA compared to WRC. For example, CBA reduces the time for decision making, reduces the frustration of the team in a collaborative design process (Arroyo et al. 2016), and is more transparent and closer to the context of the decision (Arroyo et al. 2014).

\subsection{Multidisciplinary Design Optimization Methods}


Multidisciplinary Design Optimization (MDO) methods formalize problem decomposition and coordination among groups working on the design of complex systems (AIAA 1991). Beginning in the 1980's building engineering teams began to couple building performance simulation with numerical methods to achieve optimal design solutions in less time than required for manual design iteration. A pioneering study to optimize building engineering systems was presented by Wright in 1986 when he applied the direct search method in optimizing HVAC systems (Wright 1986). Subsequently, the number of international MDO studies in the field of building science has increased exponentially (Nguyen et al. 2014). Application of these methods has been demonstrated to achieve significant improvements in building life-cycle performance compared to conventional methods (Riccardi 2011).

\section{RESEARCH METHOdOLOGY}

This study is based on a charrette experiment where a group of practitioners were required to make a decision using the WRC and CBA methods in the context of a large design space generated by MDO methods. Figure 1 depicts the main steps of this experiment.

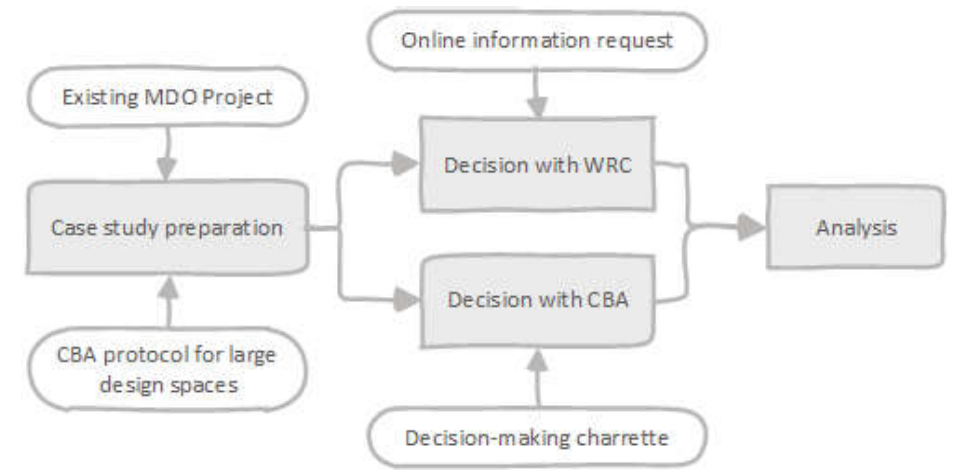

Figure 1: Main steps of the research experiment.

The case study was prepared based on an existing MDO project from where the researchers selected three decision factors plus the capital cost. The project is a hotel in Orlando, Florida, USA. The Center for Integrated Facility Engineering (CIFE) at Stanford University applied MDO methods to generate the design space based on the following design variables: building's shape factor (from 1.5 to 1.8), percentage of glazed surface per facade (from $60 \%$ to $80 \%$ ), 12 different types of glasses, 12 different types of glass insulation, and 6 options for indoor finishes. The decision factors that this research used for the CBAWRC comparison were the following:

- VOC emissions: measures the volatile organic compounds in the air, and it is a measure for the indoor air quality.

- Acoustic Reverb: measures the persistence of a sound once it is emitted by the source issuer.

- Energy: corresponds to the energy used to operate the building.

- Cost: corresponds to the capital cost of the hotel. 
The results of the MDO project allowed the researchers to determine the range for each decision factor as shown in Table 1.

Table 1: Factor's range

\begin{tabular}{cc}
\hline $\begin{array}{c}\text { Factors } \\
\text { [units] }\end{array}$ & Range \\
\hline VOC emissions & \\
{$[\mu \mathrm{g} / \mathrm{m} 3]$} & $38-126$ \\
Energy & \\
{$[$ KWh/year $]$} & $1687-7675$ \\
Acoustic Reverb & \\
{$[$ ms (T60) $]$} & $75-169$ \\
Cost & \\
{$[$ US Dollars $]$} & $6,781-75,597$ \\
\hline
\end{tabular}

One thousand design alternatives were generated for the purpose of the experiment. The case study preparation also included the application of a CBA protocol for clustering the large number of design alternatives into 10 representative design options that were used during the decision-making charrette.

The CBA method was implemented in a design charrette environment (controlled experiment with a group of people) where 11 practitioners from the AEC industry participated in the CBA decision-making process. These practitioners used CBA to individually assess the representative design solutions and then the researchers extrapolated this assessment to evaluate the IoA (importance of alternatives) for the entire design space (1000 design options). It is important to note that the cost is not used as a decision factor within the CBA method but it is used to assess the willingness to pay for the IoA of the design alternatives. Therefore, at the end of the decision-making charrette, the practitioners made a final decision based on the trade-off between the IoA of each alternative and the capital cost of that alternative.

Before the charrette, the same practitioners were asked to weigh the factors involved in the decision (including the cost) based on their perception of the relevance of each factor. These weights allowed the researchers to calculate the chosen alternative from the WRC process. This calculation consisted in assigning a relative importance for each factors on a one to ten scale with ten being the most important and one being the least important. A linear regression between these two values yielded the rating of the intermediate alternatives. Finally, the weight of each factor is multiplied by the value of the alternative, generating the final score of all the alternatives. The alternative with the highest score was the best alternative for the WRC method.

At the end of the decision-making charrette, the practitioners decided which of the two alternatives, the one chosen with WRC or the one chosen with CBA, was the alternative that provides them more benefits.

\section{RESULTS}

One of the analyses performed with the experiment data was the contrast of the WRC solution against the whole design space regarding its IoA (calculated with the 
extrapolation of the CBA results) and its capital cost. The IoA vs. Cost graphs showed two possible outcomes: the first, where the alternative selected by WRC was in the Pareto's optimal frontier; and the second case, where the WRC alternative wasn't at this curve (see Figures 2 and 3). The Pareto's optimal curve means that a decision maker can not get an alternative with a better IoA without having a higher cost. For example, in figure 3, alternative P1 is in the Pareto's optimal curve because it does not exist any alternative that has a better IoA, i.e. higher score in y axes, with an equal or lower cost, i.e. located at the left side of alternative $\mathrm{P} 1$ in the graph. If a decision maker wants to have an alternative with more IoA could chose alternative P2, which is also in the Pareto's optimal curve and it has more IoA than alternative P1, but has a higher cost over alternative P1.

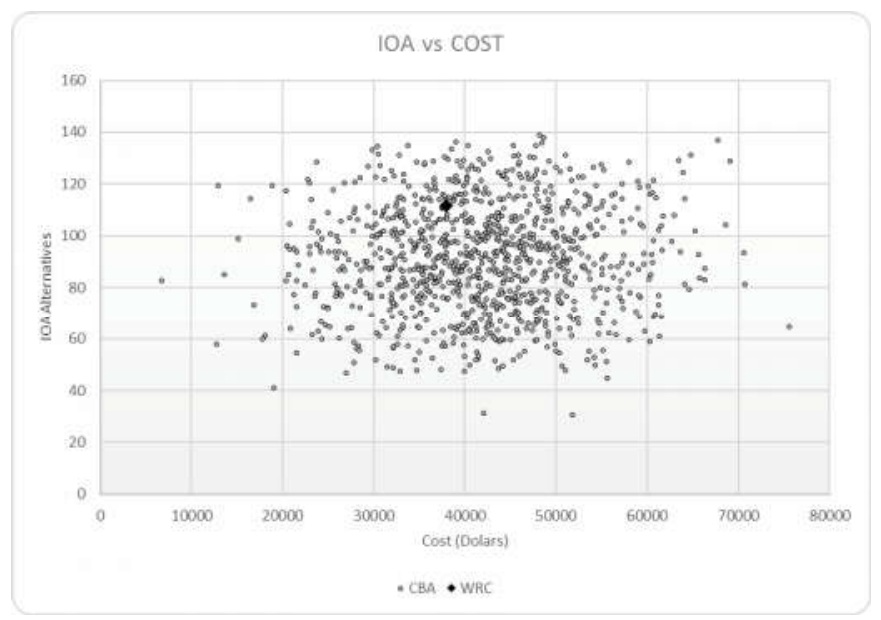

Figure 2: WRC alternative outside Pareto's optimal curve.

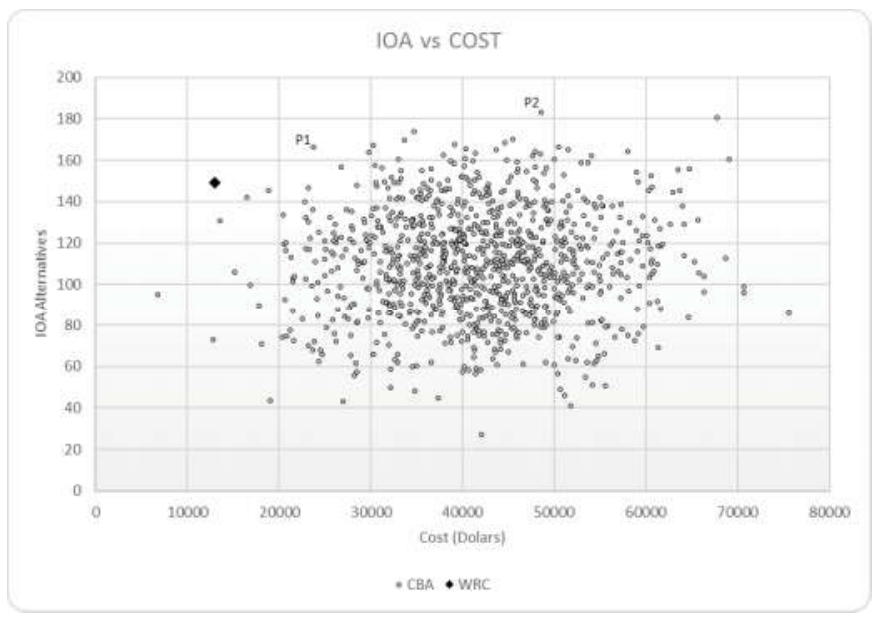

Figure 3: WRC alternative in Pareto's optimal curve.

The experiment resulted in seven practitioners selecting a Pareto optimal solution using the WRC method, and four practitioners selecting an non-Pareto optimal solution. Using the CBA method, all of the practitioners that obtained a WRC alternative that wasn't Pareto optimal selected a superior alternative. That is to say that the alternative had more IoA with the same cost than the WRC alternative or had less cost with the same IoA. Out of the seven cases where the WRC solution was Pareto optimal, five practitioners selected an alternative different from the WRC solution. 
After the CBA application, practitioners were asked to make the final decision about what of the two chosen alternatives (WRC or CBA result) would they choose. In two cases, the WRC and CBA alternatives were the same. For the nine remaining cases, the CBA alternative was preferred to the WRC alternative in all but one case. Table 2 shows the details of the decisions.

Table 2: Experiment's results

\begin{tabular}{cccc}
\hline Practitioners & $\begin{array}{c}\text { WRC alternative ubicated } \\
\text { in Pareto's optimal curve }\end{array}$ & $\begin{array}{c}\text { WRC decision is } \\
\text { different from CBA } \\
\text { decision }\end{array}$ & Final decision \\
\hline P1 & Yes $^{*}$ & Yes & Not Answer \\
P2 & Yes $^{*}$ & Yes & CBA \\
P3 & Yes $^{*}$ & Yes & CBA \\
P4 & No & Yes & CBA \\
P5 & Yes & No & Same alternative \\
P6 & Yes & Yes & CBA \\
P7 & Yes & Yes & WRC \\
P8 & No & Yes & CBA \\
P9 & Yes & No & Same alternative \\
P10 & No & Yes & CBA \\
P11 & No & Yes & CBA \\
\hline
\end{tabular}

*In those cases, WRC alternative is in Pareto's optimal curve. However, an alternative with less cost and a little difference in IoA exist (see Figure 4).

After the experiment, practitioners were asked to complete a survey about WRC and CBA methods. From that survey, all the practitioners indicated that CBA was a more transparent method than WRC. Some of the reasons were: (1) "CBA is more comprehensive. I was able to take a more informed decision"; (2) "Justifying in terms of differences between alternatives is better than a global ranking"; and, (3) "because it actually describes the advantage in an analytical way".

All practitioners indicated that in the case that they have to make a decision from a set of alternatives in the future, they will use CBA method instead of WRC. A few excerpts of their rationale included: (1) "because for decision is important analyzing the advantage in order to demonstrate to the stakeholders the benefits. It provides a clearer rationale for decision making"; and (2) "It shows more consistency to explain the importance of that particular decision you have to take in that moment".

\section{Discussion}

There are cases where WRC alternative is in Pareto's optimal curve, but exist an alternative that has a better trade off between the IoA and cost, which makes it be a better alternative for some decision makers. For example, in Figure 4 exist the alternative P3, which has slightly less IoA than WRC alternative, but has a huge reduction in cost. 
This better alternative could not be assessed in WRC method, because this method incorporate cost in the weight of factors and doesn't made an analysis of factors that are not cost versus cost, as CBA does. This final step of CBA, where the IoA and cost are evaluated for final decision, is a benefit of the method, because as practitioners indicates, this method allow to contrast the differences between alternatives and also allow to contrast more information.

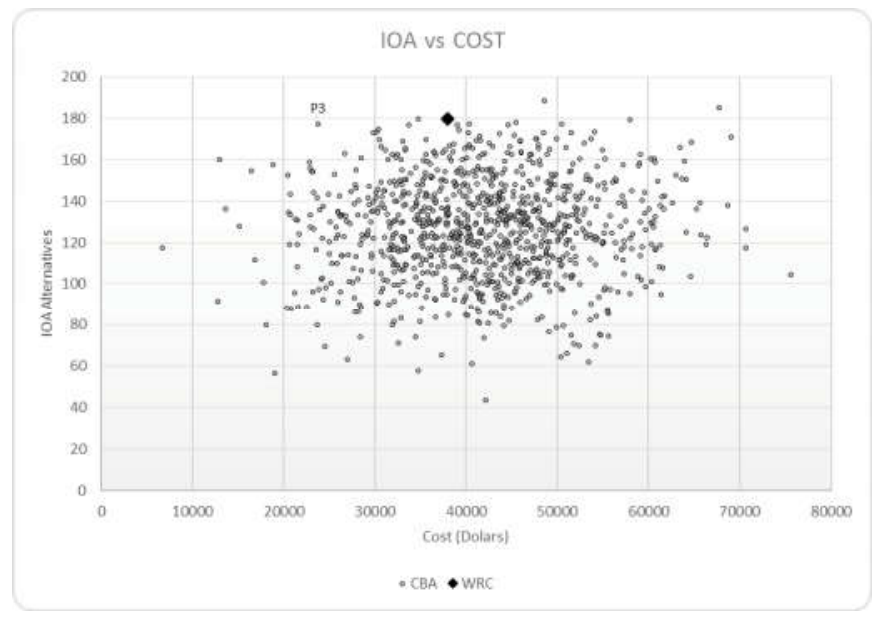

Figure 4: WRC alterative in Pareto's optimal curve, but exist better alternatives.

Most of the practitioners chose a CBA alternative different from WRC's one, even if WRC was in Pareto's optimal curve. Also, the CBA alternatives were generally higher performing and/or more economical than alternatives selected by WRC. These results demonstrate that that CBA method enabled decision makes to explicitly evaluate performance versus cost which lead to more transparent and Pareto optimal decisions considering all alternatives in the design space.

\section{CONCLUSIONS}

This study shows that it is possible to obtain better alternatives when CBA is applied to large design spaces compared to the WRC method. The benefits of the CBA method over the WRC method are obtained from the analysis of the IoA versus cost, because it is possible to comprehensively assess all the alternatives in the design space. Also, CBA makes it possible to compare the benefits of all the alternatives instead of obtaining just one alternative as the best one, as the WRC method does.

\section{REFERENCES}

AIAA (1991). Current state of the art in multidisciplinary design optimization. American Institute for Aeronautics and Astronautics Inc. MDO Technical Committee.

Arroyo, P., Fuenzalida, C., Albert, A., and Hallowell, M. (2016). Collaborating in decision making of sustainable building design: an experimental study comparing CBA and WRC methods. Energy and Buildings. doi: 10.1016/j.enbuild.2016.05.079.

Arroyo, P., Tommelein, I.D., and Ballard, G. (2015). Comparing AHP and CBA as Decision Methods to Resolve the Choosing Problem in Detailed Design. Energy Construction Engineering and Management., 141(1), p.4014063. 
Arroyo, P., Tommelein, I.D., and Ballard, G. (2014). Comparing Weighting Rating and Calculating vs Choosing By Advantages to make design choices, 1(510), pp.401-412.

Balling, R., and Rawlings, M.R. (2000). Collaborative optimization with disciplinary conceptual design. Structural and Multidisciplinary Optimization., 20(3), pp. 232-241.

Díaz, H., Alarcón, L.F., Mourgues, C., and García, S. (2017). Multidisciplinary Design Optimization through process integration in the AEC industry: Strategies and challenges. Automation in Construction.

Flager, F., Welle, B., Bansal, P., Soremekun, G., and Haymaker, J. (2009). Multidisciplinary process integration and design optimization of a classroom building. Information Technology in Construction., 14.

Flager, F., Adya, A., and Haymaker, J. (2009). AEC multidisciplinary design optimization: Impact of high performance computing. Center for Integrated Facility.

Hopfe, C.J., Augenbroe, G.L., and Hensen, J.L. (2013). Multi-criteria decision making under uncertainty in building performance assessment. Building and environment., 69, pp. 81-90.

Nguyen, A.T., Reiter, S., and Rigo, P. (2014). A review on simulation-based optimization methods applied to building performance analysis. Applied Energy., 113, 1043-1058.

Riccardi, A. 2011. Multidisciplinary design optimization for space applications. $\mathrm{PhD}$ Diss., University of Bremen, Bremen.

Sobieszczanski-Sobieski, J. (1987). The case for aerodynamics sensitivity analysis. NASA CP-2457, pp. 77-96.

Suhr, J. (1999). The Choosing by Advantages Decisionmaking System. Westport: Quorum Books.

Tappeta, R.V., and Renaud, J.E. (2001). Interactive multiobjective optimization design strategy for decision based design. Journal of Mechanical Design., 123(2), pp. 205-215.

Vandenbrande, J., Grandine, T., and Hogan, T. (2006). The search for the perfect body: Shape control for multidisciplinary design optimization. 44th AIAA Aerospace Science Meeting and Exhibit. Reno, NV, American Institute of Aeronautics and Astronautics, INC. AIAA 2006-928, pp. 1-16.

Wright, J.A. (1986). The optimised design of HVAC systems. PhD Diss., Loughborough University, Loughborough. 